\title{
Article
}

\section{Forecasting Events in the Chaotic Dynamics of a Fiber Laser}

\author{
Andrés Aragoneses ${ }^{1,2} \mathbb{D}$, Yingqi Ding ${ }^{2}$ \\ 1 Department of Physics, Eastern Washington University, Cheney, 99004, WA; aaragoneses@ewu.edu \\ 2 Department of Physics and Astronomy, Carleton College, Northfield, 55057, MN; dingy@carleton.edu \\ * Correspondence: aaragoneses@ewu.edu; Tel.: +1-509-359-7469
}

\begin{abstract}
Being able to forecast events is of great importance in many fields, from brain behavior to earthquakes or stock markets. Because each dynamical system has intrinsic features, different statistical tools have to be used for each system. Here we study the time series of the output intensity of a fiber laser with an ordinal patterns analysis, and we look for temporal correlations in order to statistically forecast the most intense events. We set two thresholds, a low one and a high one, to distinguish between low intensity versus high intensity events. We find that when the time series is performing events below the low threshold it shows some preferred temporal patterns before performing events above a high threshold.
\end{abstract}

Keywords: forecasting; complex dynamics; fiber laser; chaos; ordinal patterns.

\section{Introduction}

Time series analysis is a widespread research field that crosses disciplines. Nature presents many dynamical systems performing complex behavior that are only accessible through the temporal evolution of one or a few of its variables. Understanding the dynamical behavior relies on finding characteristic features that allow us to classify them and make predictions about their immediate future, based on their present demeanor [1-12]. Complex systems constitute a challenge, as the nonlinear interplay between the numerous elements of the system, the presence of time delay, and noise make them impractical for a bottom-up analysis.

Lasers constitute a great example of a dynamical system performing complex behavior. Besides the innumerable technological applications, lasers are a fascinating tool for the study and characterization of nonlinear dynamics from a laboratory-controlled manner [13].

One particular case of laser manifesting complex dynamics are fiber lasers with optical feedback [14]. In previous studies $[15,16]$ it was found that behind the high complexity of the time series of the output intensity of a fiber laser, the system showed some structure that could be unveiled using an ordinal patterns analysis. It was found that the laser executes some preferred intensity and temporal patterns, and those patterns helped to detect a laminar to turbulent transition as the pump power of the laser was increased [14,15]. In Ref. [16], through a simple mathematical model and an ordinal patterns analysis, it was also seen that the transition was related to internal frequencies of the dynamics. In this paper we use the ordinal patterns analysis to study the complex behavior of the output intensity of the fiber laser, to forecast when the laser is changing from emitting low intensity to emit high intensity light.

\section{Forecasting method}

The ordinal patterns analysis method (OPAM) introduced by Bandt and Pompe in 2012 [17] studies correlations between consecutive events. It defines patterns by comparing the values of $n$ consecutive events in a time series (either magnitudes or time intervals between events), and computes the probabilities of each of the $n$ ! possible patterns. For a purely stochastic process, where no correlations exist between events, one would expect to find all patterns equally probable $(P=1 / n !)$, 
while for systems that present some intensity or temporal correlation one would expect some structure in the probabilities of the patterns. This method has been used to characterize complex dynamics based on the patterns probabilities $[9,12,18,19]$, to distinguish between stochasticity and determinism [20], or to identify transitions in the dynamics of complex networks [21]. In a recent paper [22] the method was used to classify events into two types, and to forecast when the system decides to change from one type of events to the other one.

The OPAM transforms the original series of $m$ events, $X=\left\{x_{1}, x_{2}, \ldots, x_{i}, \ldots\right\}$, into a series of $m-n$ patterns. To do so, it considers $n$ consecutive events, assigns them correlative integer numbers $(0,1$, $2, \ldots, n)$, and then orders them from smaller to larger value of the event. For example for dimension $n=2$, if $x_{i}<x_{i+1}$, it labels that pattern as 01 , if $x_{i+1}<x_{i}$, it labels that pattern as 10 . For dimension $n=3$ we have six possible patterns $(012,021,102,120,201,210)$, i.e., if $x_{i}<x_{i+1}<x_{i+2}$ it labels that pattern as $012 ; x_{i}<x_{i+2}<x_{i+1}$ is $021 \ldots$

This can be applied to the actual values of the events $(\{x(i)\})$, the magnitudes in the time series, but it can also be applied to the time intervals between events $(\{t(i+1)-t(i)\})$. The former would give us information about intensity correlations among consecutive events, while the latter about temporal correlations.

For the fiber laser under study both, intensity and temporal correlations were found (see Fig. 2a,b from Ref. [15]), where some patterns where more likely to happen in the time series. The fact that the OPs are not equally probable means that the dynamics is not compatible with a stochastic process, but it is clearly deterministic. The system showed a higher degree of determinism at the laminar-turbulent transition.

\section{Results}

We analyze the output intensity of a fiber laser with optical feedback. Time series are recorded for $625 \mathrm{~ms}$, with $5 \times 10^{7}$ points and $\Delta t=12.5 \mathrm{ps}$. The time series are recorded for output powers between $0.80 \mathrm{~W}$ and $1.50 \mathrm{~W}$ (see Ref. [14] for details). The time series are subtracted the average and normalized to the standard deviation. We detect the peaks in the time series as events and study the time series of these events.
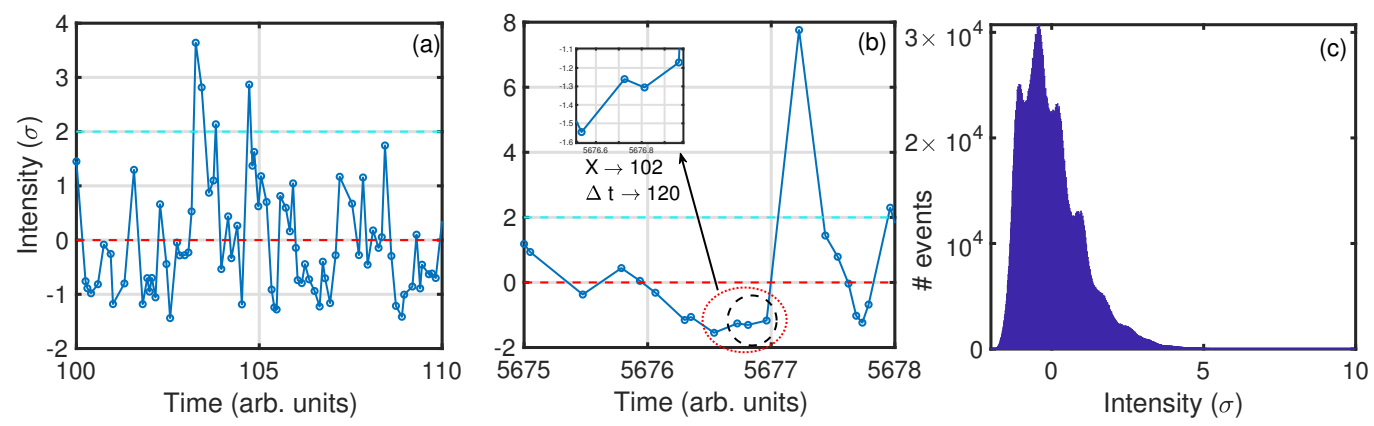

Figure 1. (a) Time series of the events that oscillate around 0 but that present some intensity events larger $2 \sigma$. Two thresholds are depicted, a low one at the mean value of the intensities, $t h_{0}=0$, and a high one at $t h^{*}=2 \sigma$. (b) Detail of the time series where four events are selected, below $t h_{0}$ that precede a high intensity event, above $t h^{*}$. The dotted red circle and the dashed black circle indicate the four (three) consecutive events below th $h_{0}$ used to generate an OP. Three consecutive events when OPs are computed with intensities, and four consecutive events when OPs are computed. (c) Histogram of events for power of $1.20 \mathrm{~W}$. The distribution of events shows some structure and a tail that reaches values larger than $6 \sigma$.

Figure 1a shows a section of a time series of events. The time series of the output intensity shows a wide distribution with most values around $\pm 2 \sigma$ and fewer events reaching values $>6 \sigma$. The insert in Fig. 1b shows four consecutive events and the corresponding OP generated. The black circle in Fig. $1 \mathrm{~b}$ indicates the three events considered to generate the OP with intensity of the values 
$\left(X_{i+1}>X_{i}>X_{i+2} \rightarrow 102\right)$, and the red circle indicates the four consecutive events considered to generate the OP with their inter-event time-intervals $\left(\Delta t_{i+1}<\Delta t_{i+2}<\Delta t_{i} \rightarrow 120\right)$.

Figure $1 \mathrm{c}$ shows the histogram of the intensity for $1.00 \mathrm{~W}$. This histogram shows a long tail for high intensities. This type of long-tail distribution is present in a wide variety of systems [23-26], that can lead to unwanted phenomena. Hence the importance of understanding and being able to forecast when the system in going to perform events corresponding to the tail, sometimes referred to as extreme events.

We define a threshold, $t h^{*}$, to classify the events as large, or extreme, when they are above that threshold. We are interested in predicting when the system changes from emitting low intensity light (lower than $t h^{*}$ ) to emit high intensity light (higher than $t h^{*}$ ).

To forecast the change from low intensity behavior to high intensity one, we impose that at least three consecutive events are below $t h^{*}$ before the system goes to emit a high intensity event (see figure 1b). We compute the ordinal patterns (OPs) of the three inter-event time-intervals right before the system changes from low to high intensity. This will indicate if there are OPs that are more/less likely to happen before the transition, and which OPs these are.

Figure 2 shows the six OPs of dimension three versus the threshold, $t h^{*}$, for three power outputs, $P=0.80 \mathrm{~W}$ (below laminar to turbulent transition), $P=0.90 \mathrm{~W}$ (at the transition), and $P=1.10 \mathrm{~W}$ (after the transition). The top row corresponds to the OPS generated with the intensity of the events. It can be observed that there are OPs that are more likely to happen and OPs that are less likely to happen, before the transition from low to high intensity. This points to some structure in the complex dynamics that manifests as a preference of patterns before performing a high intensity event. The gray region corresponds to the probabilities of an stochastic process of the six OPs equally probable. This region depends on the number of events in each threshold. For high enough threshold the number of high intensity events preceded by low intensity events is too low to have conclusive statistics, and therefore the OPs probabilities lie in the gray region.

The second row in Fig. 2 groups the six OPs into three, depending on the last event, to highlight this last event (the event preceding the high intensity event) with respect to the previous two $(X X 0=$ $120+210$, the latter event is smaller that the previous two; $X X 1=021+201$, the latter event is intermediate; $X X 2=012+102$, the latter event is larger than the previous two). This indicates that between $40 \%$ and $50 \%$ of the high intensity events will be preceded by pattern $X X 2$, i.e., of the three preceding events, the latest one is higher than the other two. In other words this indicates that the change from low intensity to high intensity is not as sharp as it could be. This figure also shows that the OPs probabilities do not depend on the threshold.

The bottom row shows the OPs generated with inter-event time-intervals. For low thresholds, $t h^{*}$, the probabilities are not compatible with a stochastic process but they are mush smaller than the ones computed with intensities. This shows that the dynamics presents less intense temporal correlations than correlations in magnitude. Also, the hierarchy is different from the top to the bottom row. Also in a previous work [15] were found temporal correlations of less relevance than intensity correlations.

There is little effect of the power output of the laser on the probabilities of the OPs. For the intensity OPs, the more likely OPs are more probable and the less likely OPs are less probable at the transition power $(0.80 \mathrm{~W})$, while for the temporal OPs, all are more equally probable, lying on or very near the gray region.

Splitting the peaks into above and below a single threshold does not separate events into those that are much larger than average (events that can be considered as extreme events) and those events around and below average. In Fig. 3 we consider two thresholds, a low one, $t h_{0}$, and a high one, $t h^{*}$. We set the lower threshold at $t h_{0}=1 \sigma$ and scan the high threshold, $2 \leq t h^{*} \leq 4$. We then calculate the OPs probabilities, (see Fig. 3).

We find that there are still preferred patterns before jumping from below th to above $t h^{*}$. The preferred OPs are the same as in Fig. 2. This is interesting as the conditions to select the events to build the OPs are not the same. In the first case it was less restrictive, and low events could be very close 


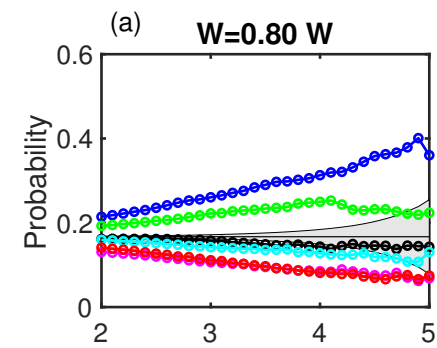

(d)

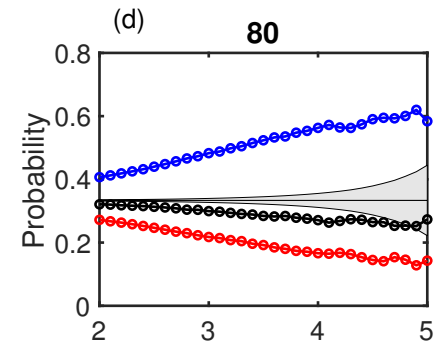

(g)

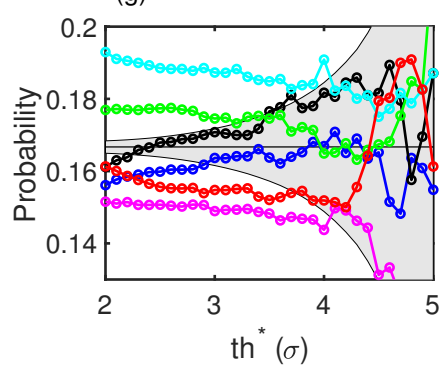

(b)

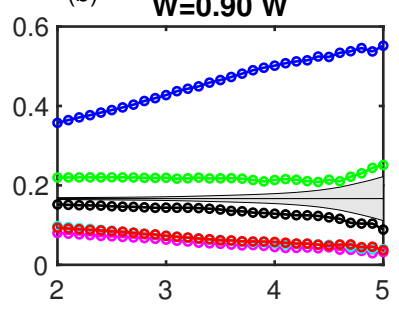

(e)

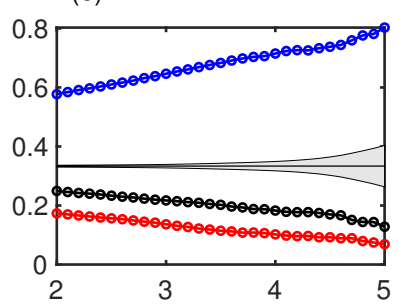

(h)

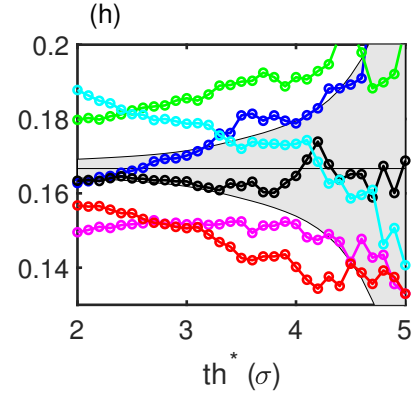

(c) $W=1.10 \mathrm{~W}$

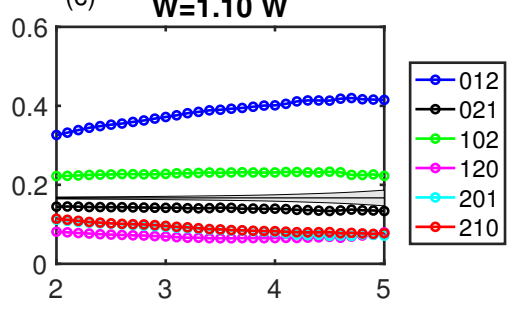

(f)

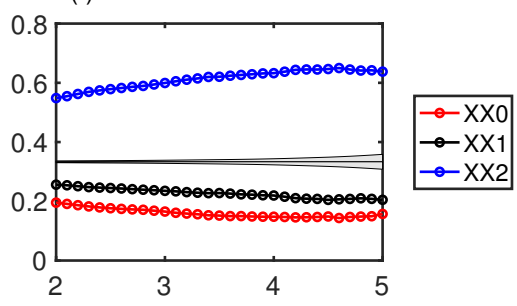

(i)

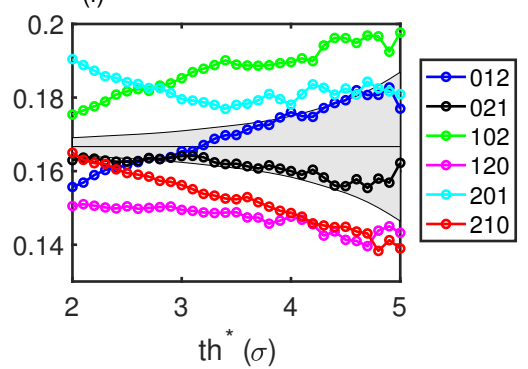

Figure 2. (a-c) Ordinal patterns computed with intensities. Three consecutive events below th*, that precede an event above $t h^{*}$ are considered for each OP. (d-f) The OPs are grouped into XX0 $=120+210$, $X X 1=021+201$, and $X X 2=012+102$. This highlights the fact that the most likely OPs to happen before a jump from low intensity events to high intensity event are those where the last event is larger than the previous two. (g-i) OPs computed with inter-event time-intervals. The gray region corresponds to a stochastic process where all six patterns are equally probable. 
to $t h^{*}$, while in the second case all events that contribute to the OP are below $t h_{0}$. This suggests that when the low events are near the low threshold, $t h_{0}$, there is more chance for a high event to happen.

With this more restrictive condition of two thresholds the number of events that satisfy that $x_{i-3}<t h_{0}, x_{i-2}<t h_{0}, x_{i-1}<t h_{0}$, and $x_{i}>t h^{*}$ is reduced. This is reflected in the wider gray regions in Fig. 3, and in the fact that above $t h^{*}=4 \sigma$ the probabilities are compatible with a stochastic process, so we cannot use this as a forecasting method. In any case, for power outputs of the laser above the transition $(P \geq 0.90 \mathrm{~W})$, and events larger than $3.5 \sigma$, we can still see that $50 \%$ of these intense events happen after the XX2 pattern.

(a)

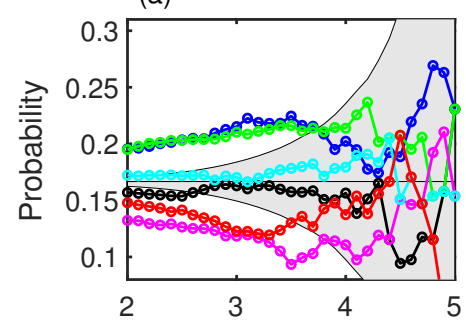

(d)

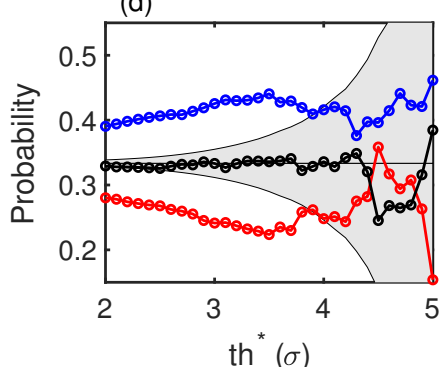

(b)

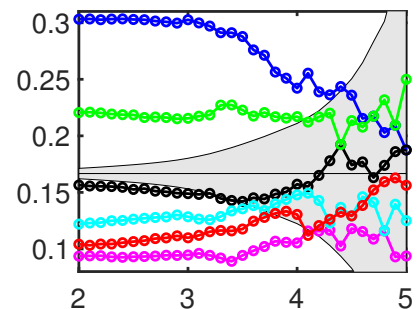

(e)

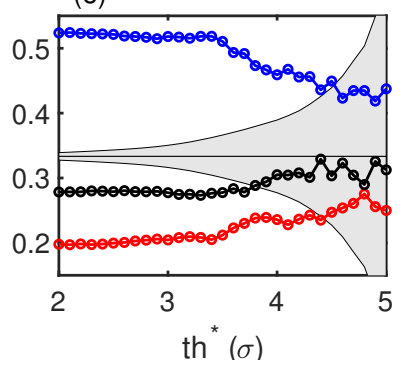

(c)

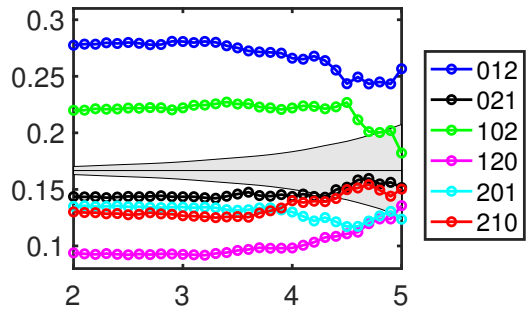

(f)

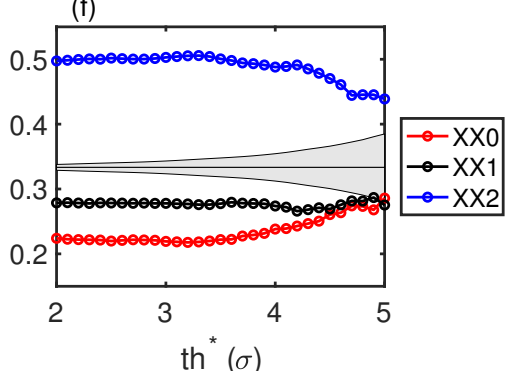

Figure 3. Ordinal patterns computed with intensities using two thresholds. A low threshold, $t h_{0}=1 \sigma$, imposes that the consecutive low events that form OPs are below $t h_{0}$. A high threshold selects the high events that take place after the OP and are above $2 \sigma \leq t h^{*} \leq 4 \sigma$. (a-c) Correspond tp the six OPs of dimension three. (d-f) correspond to the OPs combinations where the last of the three event is larger, in between, or shorter than the other two events. This more restrictive condition reduces the number of events and widens the gray area.

\section{Conclusions}

We have studied the dynamics of the output light of a fiber laser with optical feedback. By using an ordinal patterns approach, we have found signatures of determinism in its dynamics that allow us to forecast extreme events, defined as events larger than a threshold $\left(t h^{*}>2 \sigma\right)$. The system presents preferred patterns before it changes from low intensity events to more extreme events. The preferred pattern before an extreme event is the one where the latter of three consecutive events below the threshold is larger than the previous two, with probabilities that reach $80 \%$ in some cases, while the less probable pattern is the one where the latter event is less intense that the previous two events, which in some cases reaches less than $10 \%$.

We can compute the patterns with the intensity of the events or with the inter-event time-intervals. The former takes advantage of intensity correlations in the system while the latter on temporal correlations, both of them present in the dynamics. In this system the more efficient way to compute the patterns for forecasting purposes is with the actual intensity of the events. For patterns computed with inter-event time-intervals the probabilities, although still not compatible with a stochastic process, are lower and therefore less efficient to forecast the more extreme events.

The fact that there is $70 \%$ higher probability of pattern XX2 before a sudden increase in intensity has to be considered carefully, as there are much less events that are higher than $t h^{*}$ than events below 
$t h_{0}$. This means that not after $70 \%$ of all patterns XX2 in the time series will take place an extreme event, but that $70 \%$ of extreme events are preceded by pattern XX2.

One interpretation for this preference for specific patterns when the system leaves the low intensity regime and explores the higher intensity regime is that there is a specific region in phase space that triggers extreme events, and those patters are the signature of that region in phase space.

This methodology can be applied to other complex dynamical systems that manifest time series of events, with a long-tail histogram.

Author Contributions: Y. D. analyzed the data, A. A. wrote the manuscript. Y. D. and A. A. discussed the results and revised the manuscript.

Funding: This work was supported in part by Carleton College Towsley Endowment.

Acknowledgments: The authors thank N. Tarasov, D. V. Churkin, and S. K. Turitsyn for the experimental data.

Conflicts of Interest: The authors declare no conflict of interest.

\section{References}

1. Robert J. Geller, David D. Jackson, Yan Y. Kagan, and Francesco Mulargia, Earthquakes Cannot Be Predicted. Science 1997, 275, 3506, 1616.

2. Álvaro Corral, Long-Term Clustering, Scaling, and Universality in the Temporal Occurrence of Earthquakes. Phys. Rev. Lett. $2004,92,10,108501$.

3. Yan Y. Kagan, Worldwide earthquake forecasts. Stoch. Environ. Res. Risk Assess. 2017,6, 1273-1290.

4. Álvaro Corral, Josep Sardanyés, and Lluís Alsedà, Finite-time scaling in local bifurcations. Scientific Reports 2018, 8, 11783.

5. Alexander B. Neiman, and David F. Russell, Models of stochastic biperiodic oscillations and extended serial correlations in electroreceptors of paddlefish. Phys. Rev. E 71, 061915 (2005).

6. Belén Sancristóbal, Beatriz Rebollo, Pol Boada, Maria V. Sánchez-Vives, and Jordi García-Ojalvo. Collective stochastic coherence in recurrent neuronal networks. Nat. Physics 2016, 12, 881-888.

7. Vasyl Palchykov, Marija Mitrovic, Hang-Hyun Jo, Jari Saramäki, and Raj Kumar Pan. Inferring human mobility using communication patterns. Scientific Reports 2014, 4, 6174.

8. C.-K. Peng, J. Mietus, J. M. Hausdorff, S. Havlin, H. E. Stanley, and A. L. Goldberger. Long-Range Anticorrelations and Non-Gaussian Behavior of the Heartbeat. Phys. Rev. Lett. 1993, 70, 9, 1343-1346.

9. U. Parlitz, S. Berg, S. Luther, A. Schirdewan, J. Kurths, N. Wessel. Classifying cardiac biosignals using ordinal pattern statistics and symbolic dynamics. Comput. Biol. Med. 2012, 42, 319-327.

10. Massimiliano Zanin, Luciano Zunino, Osvaldo A. Rosso, and David Papo, Permutation Entropy and Its Main Biomedical and Econophysics Applications: A Review. Entropy 2012, 14, 1553-1577.

11. Miguel C. Soriano, Jordi García-Ojalvo,Claudio R. Mirasso, and Ingo Fischer Complex photonics: Dynamics and applications of delay-coupled semiconductors lasers. Rev. Mod. Phys. 2013, 85, 421(50).

12. Luciano Zunino, Massimiliano Zanin, Benjamin M. Tabake, Darío G. Pérez, Osvaldo A. Rosso. Forbidden patterns, permutation entropy and stock market inefficiency. Physica 2009, 388, 2854-2864.

13. Miguel C. Soriano, Jordi García-Ojalvo, Claudio R. Mirasso and Ingo Fischer. Complex photonics: Dynamics and applications of delay-coupled semiconductors lasers. Rev. Mod. Phys 2013, 85, 412.

14. E. G. Turitsyna, S. V. Smirnov, S. Sugavanam, N. Tarasov, X. Shu, S. A. Babin, E. V. Podivilov, D. V. Churkin, G. E. Falkovich, and S. K. Turitsyn. The laminar?turbulent transition in a fibre laser. Nat. Photonics 2013, 7, $783,2864$.

15. Andrés Aragoneses, L. Carpi, N. Tarasov, D. V. Churkin, M. C. Torrent, C. Masoller, and S. K. Turitsyn. Phys. Rev. Lett 2016, 116, 033902.

16. L. Carpi and C. Masoller. Persistence and stochastic periodicity in the intensity dynamics of a fiber laser during the transition to optical turbulence. Phys. Rev. A 2017, 97, 023842.

17. J. M. Amigo, K. Keller, and J. Kurths, Recent progress in symbolic dynamics and permutation complexity: ten years of permutation entropy. Eur. Phys. J. Spec. Top. 2013, 222, 2. 
18. O.A. Rosso, and C. Masoller. Detecting and quantifying temporal correlations in stochastic resonance via information theory measures. Eur. Phys. J. B, 2009, 69, 37-43.

19. Max L. Trostel, Moses Z. R. Misplon, Andrés Aragoneses, and Arjendu K. Pattanayak. Characterizing Complex Dynamics in the Classical and Semi-Classical Duffing Oscillator Using Ordinal Patterns Analysis. Entropy, 2018, 20, 40.

20. Andrés Aragoneses, Nicolás Rubido, Jordi Tiana-Alsina, M. C. Torrent, and Cristina Masoller, Distinguishing signatures of determinism and stochasticity in spiking complex systems. Scientific Reports 2013, 3, 1778.

21. Jiayang Zhang, Jie Zhou, Ming Tang, Heng Guo, Michael Small, and Yong Zou, Constructing ordinal partition transition networks from multivariate time series. Scientific Reports 2017, 7, 7795.

22. Meritxell Colet and Andrés Aragoneses, Forecasting Events in the Complex Dynamics of a Semiconductor Laser with Optical Feedback. Scientific Reports 2018, 810741.

23. Hugo L. D. de S. Cavalcante, Marcos Oria, Didier Sornette, Edward Ott, and Daniel J. Gauthier. Predictability and Suppression of Extreme Events in a Chaotic System. Phys. Rev. Lett. 2013, 111, 198701.

24. Alexander N. Pisarchik, Rider Jaimes-Reátegui, Ricardo Sevilla-Escoboza, G. Huerta-Cuellar, and Majid Taki. Rogue Waves in a Multistable System. Phys. Rev. Lett. 2017, 107, 274101.

25. Jordi Zamora-Munt, Bruno Garbin, Stephane Barland, Massimo Giudici, Jose R. Rios Leite, Cristina Masoller, and Jorge R. Tredicce. Rogue waves in optically injected lasers: Origin, predictability, and suppression. Phys. Rev. Lett. 2013, 87, 035802.

26. Tian Jin, Chen Siyu, and Cristina Masoller. Generation of extreme pulses on demand in semiconductor lasers with optical injection. Opt. Expr. 2017, 35, 31326. 